\title{
A fingerprint spoof detection based on MLP and SVM
}

\author{
Luis Filipe A. Pereira, Hector N. B. Pinheiro, Jose Ivson S. Silva, Anderson G. Silva, Thais M. L. Pina, \\ George D. C. Cavalcanti, Tsang Ing Ren and Joao Paulo N. de Oliveira \\ Itautec Labs, Center of Informatics - Federal University of Pernambuco, Recife, Brazil \\ \{lfap, hnbp, jiss, ags, tmlp, gdcc, tir, jpno\}@ cin.ufpe.br
}

\begin{abstract}
We introduce a fingerprint spoof detection technique based on MLP and SVM that combines several features. The proposed technique is evaluated on two scenarios: (i) when an impostor can perform consecutive attempts to be considered authentic; and, (ii) when the system deals with fingerprints from elderly people. In order to analyze these scenarios, a database was developed. The results show that the proposed combination of features increases the system performance in at least $33.56 \%$ and that the average error increases as more attempts for acceptance are allowed. The SVM classifier presents better performance in almost all the tested configurations. However, MLP is more accurate with biometrics from elderly people.
\end{abstract}

\section{INTRODUCTION}

According to Roberts [1], there are eighteen points at which a biometric system can be attacked. Attacks like communication intersection between system inner modules or addition of information on database require a high level of system technical knowledge. On the other hand, providing to the sensor a fake physical biometric is an easier way to overtake the system security. Fingerprints, in particular, can be spoofed from common materials, such as silicone and wood glue [2], [3]. Therefore, a safe fingerprint system must distinguish correctly a spoof from an authentic finger. Software-based solutions focused on computer vision can detect a biometric spoof from analysis of fingerprint features in a captured image.

Several features for fingerprint spoof detection have been proposed in the literature. Kim et al. [4] classified them into three types according to the investigated properties: physiologic perspiration phenomenon [5], [6], [7], skin distortion [8] and quality measures [9], [10]. Marasco et al. [11] classified the features in three different image processing areas: signal processing methods [5], [9], first order statistics [12] and color intensity levels [7]. Galbally et al. [2] proposed a classification that differentiates the fingerprint image quality measures used to detect spoofs. They focused on fingerprint ridges which create patterns on images. Each quality index can measure: ridge strength [13], [14], ridge continuity [13], [15] or ridge clarity [15], [16], [17].

The methods proposed by Abhyankar et al. [6] and Moon et al. [9] performed a spoof detection without any machine learning algorithm. They compared the final score of the evaluated feature with a threshold previously defined. Antonelli et al. [8] aligned the features data sequence of a new image with a real skin distortion reference sequence using Dynamic Time
Warping to evaluate the level of similarity between data. Tan et al. [7] used a Decision Tree to evaluate a set of features and detect spoof fingerprints. Derakhshani et al. [5] used a Back Propagation Neural Network to classify the patterns. Galbally et al. [10] used a Linear Discriminant Analysis as classifier. Marasco et al. [11] achieved better results with a Multilayer Perceptron and a Decision Tree applied to different databases.

In this paper, we propose a technique to perform fingerprint spoof detection and an approach to evaluate the system security when consecutive attempts are allowed for acceptance. The proposed approach automatically selects the best features from the set of features used by Marasco et al. [11] and Galbally et al. [10]. Two machine learning techniques are used to classify patterns: Multilayer Perceptron and Support Vector Machine. Since the public databases, Fingerprint Liveness Detection Competition 2009 [18] and 2011 [19], do not have enough information about volunteers whose contributed with their fingerprints, a database was developed. This database comprises consecutive attempts tests and fingerprints from elderly people. An important subset of biometric system users is formed by elderly people. Their old skin usually provides low quality images which can lead to system errors. The behavior of the proposed system is also evaluated over these group of volunteers.

This paper is organized as follows. The architecture of the proposed fingerprint spoof detection system is described in Section II. Section III presents the experimental study and Section IV shows the conclusions.

\section{Proposed ARCHIteCture}

The architecture of the proposed fingerprint spoof detection system is shown in Fig. 1. For a given fingerprint image, the Feature Extraction module calculates a vector of characteristics that describes the image. The Feature Selector module searches for the subset of the most representative features. The Classifier aims to discriminate between live and spoof fingerprints. The next subsections details each module.

\section{A. Feature Extraction}

For a given fingerprint image, the feature vector $\mathbf{x}$ which describes the biometrics is generated by the feature extraction procedure as defined below in Equation (1): 


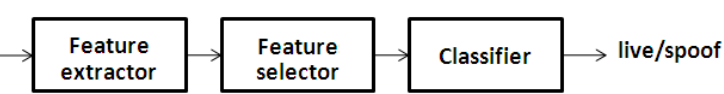

Fig. 1. Architecture of the proposed fingerprint spoof detection

$$
E(x(i, j))=\mathbf{x}=\left[\begin{array}{c}
c_{1} \\
c_{2} \\
\cdots \\
c_{n}
\end{array}\right]
$$

where $x(i, j)$ is a fingerprint image and $E$ is a set of feature extraction functions. $E=\left\{E_{1}, E_{2}, \ldots, E_{n}\right\}$ where $n$ represents the size of the feature vector, thus $E_{k}(x(i, j))=$ $c_{k}, k=1,2, \ldots, n$. Each element $c_{k} \in \mathbf{x}$ is a real number that quantifies the $k^{t h}$ feature of the vector.

In this work, the size of the feature vector is equal to seventeen $(n=17)$. Each one of these features are defined below:

- The features used by Marasco et al. [11] are useful because they can lead the fingerprint spoof detection module to a better performance than the best algorithm evaluated on Liveness Detection Competition 2009 [18]. Since no information about the algorithms evaluated on Liveness Detection Competition 2011 [19] are available, no performance study was conducted with them. These features are based on signal processing, first order statistics and gray level intensity and they are defined as follows:

\section{- Signal processing.}

* $\mathbf{E}_{1}$ : Individual pore spacing feature: through the analysis of pixels along the ridges, it is possible to find sweat pores in the image. Live fingerprint images fingers should have these information strongly defined. The power of signal in sweat pores frequency (11 to 33) [5] is defined as:

$$
\begin{gathered}
c_{1}=\sum_{k=11}^{k=33}\left(\frac{\sum_{i=1}^{n}\left\|F\left(S_{i}^{\prime}(p)\right)\right\|}{n}\right)^{2} \\
S_{i}^{\prime}=S_{i}-\operatorname{mean}\left(S_{i}\right)
\end{gathered}
$$

where $n$ is the total number of individual ridges in a fingerprint image, $S_{i}$ is the one-dimensional gray level signal along the $i_{t h}$ ridge and $F(x)$ the Fourier Transform.

* $\mathbf{E}_{2}$ : Residual noise feature: the coarseness of the surface of the sample can be measured as the total amount of Gaussian white noise in the original image [9]. An additional effort has to be done to produce fingerprint spoofs as coarse as live fingers. The amount of noise in $x(i, j)$ can be evaluated as:

$$
c_{2}=\operatorname{std}\left(x(i, j)-x^{\prime}(i, j)\right)
$$

where $x^{\prime}(i, j)$ is the denoised image using the wavelet based approach [20] and std is the standard deviation function.

- First order statistics. These features measure the visual differences which can be observed in the live and spoof fingerprints [12].

$* \mathbf{E}_{3}$ : Energy feature:

$$
c_{3}=\sum_{n=1}^{N-1} H(n)^{2}
$$

$* \mathbf{E}_{4}$ : Entropy feature:

$$
c_{4}=-\sum_{n=1}^{N-1} H(n) \log H(n)
$$

* $\mathbf{E}_{5}$ : Mean feature:

$$
c_{5}=\frac{\sum_{n=1}^{N} H(n)}{N}
$$

* $\mathbf{E}_{6}$ : Variance feature:

$$
c_{6}=\sum_{n=1}^{N}(n-\mu)^{2} H(n)
$$

$* \mathbf{E}_{7}$ : Skewness feature:

$$
c_{7}=\frac{1}{\sigma^{3}} \sum_{n=1}^{N-1}(n-\mu)^{3} H(n)
$$

* $\mathbf{E}_{8}$ : Kurtosis feature:

$$
c_{8}=\frac{1}{\sigma^{4}} \sum_{n=1}^{N-1}(n-\mu)^{4} H(n)
$$

* $\mathbf{E}_{9}$ : Variation coefficient feature:

$$
c_{9}=\frac{\sigma}{\mu}
$$

where $H(n)$ is the equalized and normalized histogram of $x(i, j), N$ is the total number of bins in the histogram, $\mu$ is the mean and $\sigma$ is the standard deviation of $H(n)$.

- Gray level intensity. Due the perspiration phenomenon, live fingers demonstrate a distinctive spatial moisture pattern which implies on gray level variations along the image [6].

* $\mathbf{E}_{10}$ : Gray level 1 feature:

$$
c_{10}=\frac{P_{150,253}}{P_{1,149}}
$$

* $\mathbf{E}_{11}$ : Gray level 2 feature:

$$
c_{11}=\frac{P_{246,256}}{P_{1,245}}
$$

where $P_{i, j}$ is the total of pixels with gray level intensity between $i$ and $j$. 
* $\mathbf{E}_{12}$ : Gradient feature:

$$
c_{12}=\text { mean }\left(\sqrt{\left(\frac{\partial x(i, j)}{\partial x}\right)^{2}+\left(\frac{\partial x(i, j)}{\partial y}\right)^{2}}\right)
$$

- Features used by Galbally et al. [2] are useful because they produce characteristics not previously investigated for fingerprint liveness detection. From the segmented image $x_{s}(i, j)$ [21], the features quantify:

- Ridge strength. These features measure the energy concentration along the image and capture fingerprint sharpness. It is expected that good quality live fingerprints produce images with well defined ridges.

* $\mathbf{E}_{13}$ : Orientation certainty level (OCL) features: it measures the energy concentration along the dominant direction of ridges [13]. An OCL index is computed for each non overlapped image block of $x(i, j)$ as the ratio between the two eigenvalues of the covariance matrix of the gradient vector. The final index is evaluated as:

$$
c_{13}=\operatorname{mean}(O \bullet W)
$$

where $O$ is the matrix with all OCL values, $W$ is a matrix of weights at which the central elements have more power and $\bullet$ is the element by element matrix multiplication operator.

* $\mathbf{E}_{14}$ : Energy concentration in the power spectrum (ECPS) feature: it evaluates the concentration of energy in the image between 0.5 and 0.06 frequencies [14]. All bandwidth is segmented into $T$ sub-bands. The final feature value is defined as:

$$
\begin{aligned}
& c_{14}=\log T-S \\
& S=\sum_{t=1}^{T} P_{t} \log P_{t}
\end{aligned}
$$

where $P_{t}$ is the total amount of energy in the $t_{t h}$ sub-band.

- Ridge continuity. The goal is to evaluate the flow of ridge curves along the fingerprint pattern. It is expected that those line curves flows smoothly in live good quality fingerprint images.

* $\mathbf{E}_{15}$ : Local orientation quality (LOQ) feature: it measures the difference in curve angle directions between neighbor non overlapped image blocks.

$$
\begin{gathered}
c_{15}=\operatorname{mean}\left(Q^{\prime}\right) \\
Q^{\prime}(i, j)=\sum_{p=-1}^{1} \sum_{q=-1}^{1}|Q(i, j)-Q(i+p, j+q)|
\end{gathered}
$$

where $Q(i, j)$ is the angle in an image block [15].

- Ridge clarity. Based on Coli et al. [16], these features are used to detect spoof fingerprint from gray level image intensity.

* $\mathbf{E}_{16}$ : Meaningful mean feature: evaluates the mean gray level intensity only from the foreground image.

$$
c_{16}=\operatorname{mean}\left(x_{s}(i, j)\right)
$$

* $\mathbf{E}_{17}$ : Meaningful standard deviation feature: measure the standard deviation gray level intensity only from the foreground image.

$$
c_{17}=\operatorname{std}\left(x_{s}(i, j)\right)
$$

\section{B. Feature selection}

Since a subset of total features can provide a better discriminant result, a Sequential Forward Selection [22] technique is used for this purpose. It performs a simple Hill-Climbing search, where the attribute that leads to the best evaluation is added to the set. The evaluation function used is the correct classification rate presented on the training dataset. This selection are made for both classifiers (MLP and SVM).

\section{Classifier}

The fingerprint spoof detection problem can be seen as a two class classification problem. Thus, a pattern $\mathbf{x}$ can be classified into authentic or spoof class by machine learning techniques as the Multilayer Perceptron (MLP) and the Support Vector Machine (SVM).

The MLP used has only one hidden layer and it is trained by the Backpropagation algorithm. The learn rate is equal to 0.6 , the momentum rate is equal to 0.2 and the training has at most 5000 epochs. The activation function used is the Sigmoid and the percentage size of the validation set is $30 \%$. The validation threshold used in the training is equal to 20 . This validation threshold dictates how many times the validation set error can deteriorate until the end of the training. The output layer is composed by two nodes which references the live and spoof classes. Beside this fact, only the response of the live class node is considered. This response is compared to a classification threshold and the respective sample is considered live if this response is greater than it.

The SVM used is trained by using the Pearson VII Universal Kernel (PUK) [23] with sigma and omega values set to 1.0. The response of the SVM is also compared to a classification threshold. All the samples whose response is greater than the threshold are considered live.

\section{EXPERIMENTS}

\section{A. Database}

The database contains 3240 images which were extracted from 23 volunteers. Seventeen of them were adult whose age 
was between 20 and 55 years old and the others were elderly people which were more than 60 years old.

The samples were organized into three groups according to their sample material: live, silicone and wood glue.

- Live: 230 samples from all fingers of each volunteer.

- Silicone: 170 samples from all fingers of all adult volunteers.

- Wood Glue: 140 samples from all fingers of only 14 of all adult volunteers.

For each group, the number of samples was duplicated by two distinct pressure levels upon the sensor surface. Finally, three consecutive similar captures of the same biometric were made to simulate the scenario where three attempts are allowed for acceptance. Resulting in a total amount of 1380 images from Live, 1020 images from Silicone and 840 images from Wood Glue.

Based on the captured images, two datasets were constructed: $D B 1$ and $D B 2$. The set $D B 1$ contains fingerprint images only from adult volunteers while the set $D B 2$ has images from adults and elderly. Both sets contains all spoof samples captured.

\section{B. Consecutive attempts}

Since the database has information of three consecutive captures from each biometrics, it is simulated a scenario where a fingerprint is accepted when is classified as live for the first time. The performance of the system is registered for one, two and three attempts. The goal is to find a mathematical approximation which indicates the system security when an impostor tries to get access many times in a row.

\section{Performance measures}

The following rates are used to evaluate the system performance:

- False Acceptance Rate (FAR): from all spoof fingerprints evaluated, it represents the rate of those classified as live;

- False Rejection Rate (FRR): from all live fingerprints evaluated, it represents the rate of those classified as spoof;

- Average Classification Error (ACE): it is the average of FAR and FRR weighted by the number of spoof and live samples, respectively.

\section{Results}

All rates showed in this section were obtained from an 8fold cross-validation procedure. As two classifiers were trained 8 times each, distinct sets of features were selected when the training set changed. The histogram in Fig. 2 shows the occurrence of each feature when the MLP and SVM are applied over $D B 1$. It is possible to observe that most of the features based on first order statistics were rarely chosen. Thus, $\mathrm{E}_{4}, \mathrm{E}_{5}, \mathrm{E}_{6}, \mathrm{E}_{7}$ and $\mathrm{E}_{9}$ are not the best features to discriminate live from spoof images.

The experiments evaluated four distinct system configurations together with each classifier:



Fig. 2. Frequency of each feature after the feature selection procedure


Fig. 3. Best threshold for each configuration using MLP: (a) configuration (i), (b) configuration (ii), (c) configuration (iii) and (d) configuration (iv)

(i) $\left\{E_{1}, E_{2}, \ldots, E_{12}\right\}$ without feature selection;

(ii) $\left\{E_{1}, E_{2}, \ldots, E_{12}\right\}$ with feature selection;

(iii) $\left\{E_{1}, E_{2}, \ldots, E_{17}\right\}$ without feature selection;

(iv) $\left\{E_{1}, E_{2}, \ldots, E_{17}\right\}$ with feature selection.

For each configuration described above, an optimal classification threshold was founded looking for the value which conduced the system to the lowest ACE rate. For single attempt tests with biometrics from $D B 1$, Fig. 3 and Fig. 4 illustrate the position of those points on ROC curves of each configuration with MLP and SVM, respectively.

The Table I explicit the Area Under Curve (AUC) of ROC curves figured in Fig. 3 and Fig. 4. The higher AUC values which are observed for SVM classifier indicates that its performance is better compared to MLP for $D B 1$ dataset.

The Table II details the system performance at each operation point indicated on Fig. 3 and Fig. 4. It can be also seen in Table II that the last two configurations, (iii) and (iv), obtained better rates than the first two, (i) and (ii). These results high- 

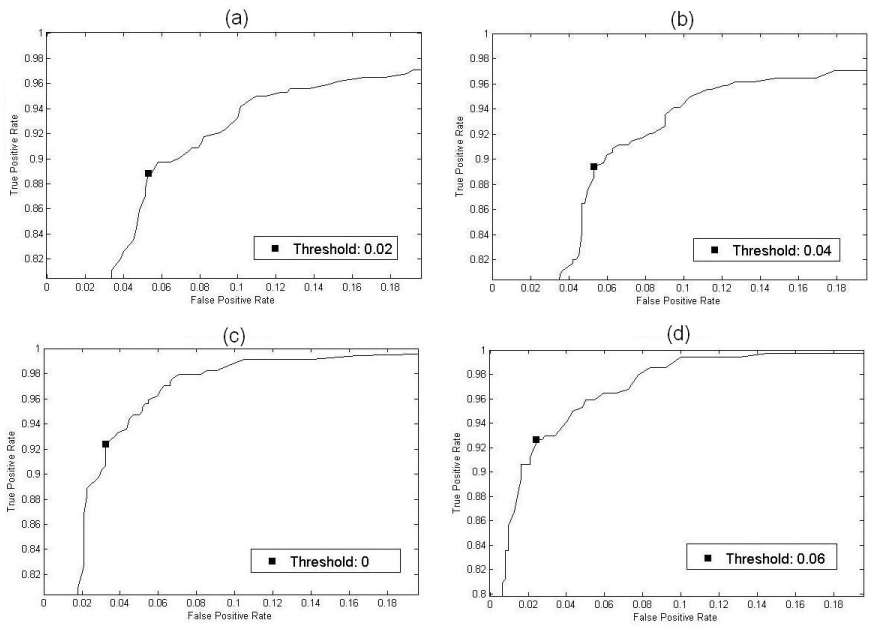

Fig. 4. Best threshold for each configuration using SVM: (a) configuration (i), (b) configuration (ii), (c) configuration (iii) and (d) configuration (iv)

TABLE I

AREA UNDER CURVE FOR EACH SYSTEM CONFIGURATION

\begin{tabular}{|c|c|c|}
\hline Configuration & Classifier & AUC \\
\hline \multirow{2}{*}{ (i) } & MLP & 0.964501 \\
& SVM & 0.967954 \\
\hline \multirow{2}{*}{ (ii) } & MLP & 0.961341 \\
& SVM & 0.969077 \\
\hline \multirow{2}{*}{ (iii) } & MLP & 0.987730 \\
& SVM & 0.990531 \\
\hline \multirow{2}{*}{ (iv) } & MLP & 0.986648 \\
& SVM & 0.990910 \\
\hline
\end{tabular}

light the discriminant power of the features $E_{13}, E_{14}, E_{15}, E_{16}$ and $E_{17}$ - these features are present in configurations (iii) and (iv) and absent in configurations (i) and (ii). These features increased the performance in at least $33.56 \%$. The feature selection procedure base on the Sequential Forward algorithm also provides a contribution to decrease system error rates, the results were better with it than the ones founded when the whole feature set was used.

TABLE II

CLASSIFICATION ERROR RATES ON A SINGLE ATTEMPT FOR ACCEPTANCE

\begin{tabular}{|c|c|c|c|c|}
\hline Configuration & Classifier & FAR (\%) & FRR (\%) & ACE (\%) \\
\hline \multirow{2}{*}{ (i) } & MLP & 9.02 & 9.39 & 9.15 \\
& SVM & 5.32 & 11.16 & 7.39 \\
\hline \multirow{2}{*}{ (ii) } & MLP & 8.22 & 10.29 & 8.95 \\
& SVM & 5.32 & 10.58 & 7.18 \\
\hline \multirow{2}{*}{ (iii) } & MLP & 3.22 & 7.61 & 4.77 \\
& SVM & 2.26 & 7.94 & 4.27 \\
\hline \multirow{2}{*}{ (iv) } & MLP & 4.52 & 3.81 & 4.27 \\
& SVM & 2.42 & 7.35 & 4.17 \\
\hline
\end{tabular}

Tables III and IV show the results for two and three attempts for acceptance. Note the downward trend on FRR while FAR increases as many attempts are allowed for the user.
TABLE III

CLASSIFICATION ERROR RATES ON TWO ATTEMPTS FOR ACCEPTANCE

\begin{tabular}{|c|c|c|c|c|}
\hline Configuration & Classifier & FAR (\%) & FRR (\%) & ACE (\%) \\
\hline \multirow{2}{*}{ (i) } & MLP & 12.73 & 6.15 & 10.40 \\
& SVM & 7.90 & 5.58 & 7.08 \\
\hline \multirow{2}{*}{ (ii) } & MLP & 11.28 & 7.34 & 9.89 \\
& SVM & 8.39 & 5.58 & 7.40 \\
\hline \multirow{2}{*}{ (iii) } & MLP & 4.67 & 3.22 & 4.15 \\
& SVM & 4.03 & 3.23 & 3.75 \\
\hline \multirow{2}{*}{ (iv) } & MLP & 5.65 & 1.46 & 4.17 \\
& SVM & 4.36 & 1.76 & 3.44 \\
\hline
\end{tabular}

TABLE IV

CLASSIFICATION ERROR RATES ON THREE ATTEMPTS FOR ACCEPTANCE

\begin{tabular}{|c|c|c|c|c|}
\hline Configuration & Classifier & FAR (\%) & FRR (\%) & ACE (\%) \\
\hline \multirow{2}{*}{ (i) } & MLP & 16.44 & 3.52 & 11.86 \\
& SVM & 7.08 & 2.94 & 8.54 \\
\hline \multirow{2}{*}{ (ii) } & MLP & 15.00 & 4.11 & 11.14 \\
& SVM & 11.13 & 3.24 & 8.34 \\
\hline \multirow{2}{*}{ (iii) } & MLP & 6.12 & 0.88 & 4.26 \\
& SVM & 5.65 & 1.46 & 4.16 \\
\hline \multirow{2}{*}{ (iv) } & MLP & 8.39 & 0.58 & 5.63 \\
& SVM & 5.97 & 1.17 & 4.27 \\
\hline
\end{tabular}

The pair, SVM and configuration (iv), obtained the best performance for a single attempt. Based on this pair, Fig. 5 shows a non-linear estimative of the system performance for many consecutive attempts. On bottom-right side of figure, the inversed triangles and circles are the FRR and the FAR, respectively. The strong downward trend of FRR points fits on a descend exponential curve. Since FAR rates increases, it is modeled with the exponential inverse function. The mathematical description of those projections are defined in Equations (22) and (23).

$$
\begin{gathered}
\operatorname{far}(m)=0.031886 \times \log (m)+0.023455 \\
\operatorname{frr}(m)=0.155577 \times 0.398917^{m}
\end{gathered}
$$

where $m$ is the number of attempts.

Fig. 5 also shows that FRR tends to zero after the $5_{t h}$ attempt and FAR is lower than $15 \%$ on the first 50 attempts. Moreover, when the number of attempts are duplicated from 50 to 100 , FAR increases only 2.21 percentile points (from $14.82 \%$ to $17.03 \%$ )

Table $\mathrm{V}$ shows the performance of the configuration (iv) in $D B 2$ with previously selected classification thresholds. Since the fingerprints from elderly people were not used in the training phase and the SVM classifier led to a higher FRR, we can conclude that MLP is better suited to generalize the concept of live fingerprint. Although, this level of live fingerprints misclassification (13.68\%) may not be acceptable for some applications. In fact, an increase of $35 \%$ with live fingerprints of elderly people on database (from $D B 1$ to $D B 2$ ) cause an increase of $259.05 \%$ of the FRR comparing with the same system configuration. More specifically, 52 of all 


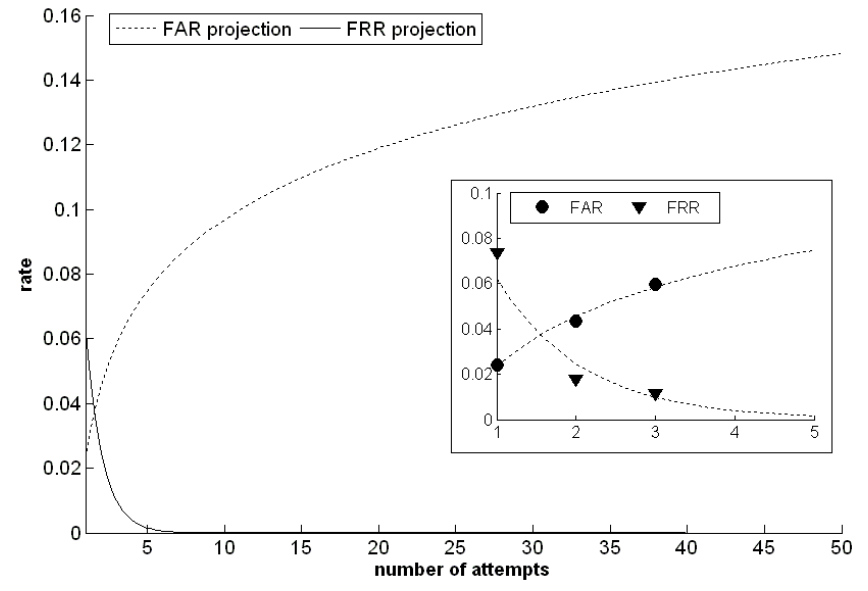

Fig. 5. Error rates projection for a high number of attempts

120 elderly people authentication attempts were refused by the spoof detection system.

TABLE V

PERFORMANCE OF THE CONFIGURATION (IV) ON DB2 FOR A SINGLE ATTEMPT FOR ACCEPTANCE

\begin{tabular}{|c|c|c|c|}
\hline Classifier & FAR(\%) & FRR(\%) & ACE(\%) \\
\hline MLP & 4.52 & 13.68 & 7.76 \\
SVM & 2.42 & 19.77 & 8.56 \\
\hline
\end{tabular}

Tables VI and VII show the results for two and three attempts. The downward trend on FRR as many attempts are allowed can decrease the misclassification of fingerprints from elderly people.

TABLE VI

PERFORMANCE OF THE CONFIGURATION (IV) ON DB2 FOR TWO ATTEMPTS FOR ACCEPTANCE

\begin{tabular}{|c|c|c|c|}
\hline Classifier & FAR(\%) & FRR(\%) & ACE(\%) \\
\hline MLP & 5.65 & 7.60 & 6.34 \\
SVM & 4.36 & 12.17 & 7.13 \\
\hline
\end{tabular}

TABLE VII

PERFORMANCE OF THE CONFIGURATION (IV) ON DB2 FOR THREE ATTEMPTS FOR ACCEPTANCE

\begin{tabular}{|c|c|c|c|}
\hline Classifier & FAR(\%) & FRR(\%) & ACE $(\%)$ \\
\hline MLP & 8.39 & 5.43 & 7.34 \\
SVM & 5.97 & 6.36 & 6.11 \\
\hline
\end{tabular}

\section{CONCLUSION}

A novel fingerprint spoof detection technique based on Multilayer Perceptron and Support Vector Machine has been proposed. This technique uses image features never combined before. Moreover, we also introduced an approach to evaluate the system performance on the scenario where several attempts are allowed for acceptance.
After extracting seventeen features from fingerprint images, the Sequential Forward Selection algorithm was used to find the most discriminant feature subset. The features based on first order statistics were not chose in most of the folds of the cross validation process. Thus, those features do not represent an interesting subset of features.

The experimental study shows that the proposed features combination increases the system performance in at least $33.56 \%$ for fingerprints of under 55 years old people. When the database contains fingerprints from elderly people, the live fingers misclassification rate is $259.05 \%$ greater. Thus, fingerprints from elderly people compound a special group where the MLP classifier obtained lower FRR rates compared to the SVM.

\section{REFERENCES}

[1] C. Roberts, "Biometric attack vectors and defences," Computers and Security, 2007, vol. 26, no. 1, pp. 14-25.

[2] J. Galbally-Herrero, J. Fierres-Aguilar, J. D. Rodriguez-Gonzalez, F. Alonso-Fernandez, J. Ortega-Garcia and M. Tapiador, "On the vulnerability of fingerprint verification systems to fake fingerprints attacks," Proceedings of Carnahan Conference Secirity Technology 40th Annual IEEE International, 2006, pp. 130-136.

[3] T. Matsumoto, H. M. K. Yamada and S. Hoshino. "Impact of artificial gummy fingers on fingerprint systems," Proceedings of SPIE. Optical security and counterfeit deterrence techniques IV, 2002, Vol. 4677, pp. 275-289.

[4] H. Kim, C. Jin and S. Elliot, "Liveness detection of fingerprint based on band-selective fourier spectrum," Information Security and Cryptology, 2007, Vol. 4817, pp. $168-179$.

[5] R. Derakhshani, S.A.C. Schuckers, L.A. Hornak, and L.O. Gorman, "Determination of vitality from a non-invasive biomedical measurement for use in fingerprint scanners," Pattern Recognition, 2003, Vol. 36, pp. 383-396.

[6] A. Abhyankar and S. Schuckers: "Integrating a wavelet based perspiration liveness check with fingerprint recognition," Pattern Recognition, 2009, 42, (3), pp. 452-464.

[7] B. Tan and S. A. C.Schuckers, S. "Liveness detection using an intensity based approach in fingerprint scanner," Proceedings of Biometrics Symposium (BSYM), 2005, Arlington, VA, pp. 19-21.

[8] A. Antonelli,R. Cappelli,D. Maio and D. Maltoni, "Fake finger detection by skin distortion analysis," Information Forensics and Security, IEEE Transactions on, Sept. 2006, vol.1, no.3, pp. 360-373.

[9] Y. S. Moon, J. S. Chen, K. C. Chan and K. C. Woo. "Wavelet based fingerprint liveness detection", Electronics Letters, 2005, vol. 41, no. 20, pp. 1112-1113.

[10] J. Galbally, F. Alonso-Fernandez, J. Fierrez and J. Ortega-Garcia. "Fingerprint liveness detection based on quality measures," Biometrics, Identity and Security (BIds), 2009, pp. 1-8.

[11] E. Marasco and C. Sansone. "An anti-spoofing technique using multiple textural features in fingerprint scanners," Biometric Measurements and Systems for Security and Medical Applications (BIOMS), 2010, pp. 8-14.

[12] A. Abhyankar and S. Schuckers. "Fingerprint liveness detection using local ridge frequencies and multiresolution texture analysis techniques," IEEE International Conference on Image Processing, 2006, pp. 321-324.

[13] E. Lim, X. Jiang and W. Yau, "Fingerprint image quality and validity analysis," Proceedings from International Conference on Image Processing, ICIP, 2002, vol. 1, pp. 469-472.

[14] Y. Chen, S. Dass and A. Jain, "Fingerprint quality indices for predicting for predicting authentication performance," Proceedings from Audio- and Video-Based Biometric Person Authentication, AVBPA, 2005, Springer, LNCS-3546, pp. 160-170.

[15] T. Chen, X. Jiang and W. Yau, "Fingerprint image quality analysis," Proceedings International Conference on Image Processing, ICIP, 2004, vol. 2, pp. 1253-1256.

[16] P. Coli, G. L. Marcialis and F. Roli, "Fingerprint silicon replicas: static and dynamic measures for vitality detection using an optical capture device," International Journal of Image and Graphics, 2008, pp. 495512 
[17] L. Hong, Y. Wan and A. Jain, "Fingerprint image enhancement: Algorithm and performance evaluation," IEEE Transactions on Pattern Analysis and Machine Intelligence, 1998, vol. 20. no 8. pp. 777-789.

[18] G. Marcialis, A. Lewicke, B. Tan and P. Coli, D. Grimberg, A. Congiu, A. Tidu, F. Roli and S. Schuckers, First International Fingerprint Liveness Detection Competition LivDet 2009.

[19] (2011, Sep.) Fingerprint Liveness Detection Competition 2011. Clarkson University and University of Cagliari. [Online]. Available: http://people.clarkson.edu/projects/biosal/fingerprint/index.php

[20] Lu, W.S.: "Wavelet approaches to still image denoising," Conf. Rec. of 31st Asilomar Conf. on Signals, Systems and Computers, November 1997, Vol. 2, pp. 1705-1709.

[21] L. Shen, A. Kot, and W. Koo, "Quality measures of fingerprint images," Proc. 3rd International Conference on Audio- and Video-Based Biometric Person Authentication, AVBPA, 2001, vol. Springer LNCS-2091, pp. 266271.

[22] M. Gutlein, E. Frank, M. Hall, A. Karwath, "Large-scale attribute selection using wrappers," Proceedings of the IEEE Symposium Series on Computational Intelligence and Data Mining (CIDM-2009), 2009, pp. 332-339.

[23] B. stn, W.J. Melssen and L.M.C. Buydens, "Facilitating the application of Support Vector Regression by using a universal Pearson VII function based kernel," Chemom. Intell. Lab. Syst., 81 (2006), pp. 29-40. 